\title{
Fate of Artificial MicroRNA-Mediated Resistance to Plant Viruses in Mixed Infections
}

\author{
Fernando Martínez, Santiago F. Elena, and José-Antonio Daròs
}

First, second, and third authors: Instituto de Biología Molecular y Celular de Plantas (Consejo Superior de Investigaciones CientíficasUniversidad Politécnica de Valencia), 46022 Valencia, Spain; second author: Santa Fe Institute, Santa Fe, NM. Accepted for publication 9 March 2013.

\begin{abstract}
Martínez, F., Elena, S. F., and Daròs, J.-A. 2013. Fate of artificial microRNA-mediated resistance to plant viruses in mixed infections. Phytopathology 103:870-876.

Artificial microRNAs (amiRNAs) are the expression products of engineered microRNA (miRNA) genes that efficiently and specifically downregulate RNAs that contain complementary sequences. Transgenic plants expressing high levels of one or more amiRNAs targeting particular sequences in the genomes of some RNA viruses have shown specific resistance to the corresponding virus. This is the case of the Arabidopsis thaliana transgenic line 12-4 expressing a high level of the amiR159-HCPro targeting 21 nucleotides in the Turnip mosaic virus (TuMV) (family

Potyviridae) cistron coding for the viral RNA-silencing suppressor HCPro that is highly resistant to TuMV infection. In this study, we explored the fate of this resistance when the A. thaliana 12-4 plants are challenged with a second virus in addition to TuMV. The A. thaliana 12-4 plants maintained the resistance to TuMV when this virus was co-inoculated with Tobacco mosaic virus, Tobacco rattle virus (TRV), Cucumber mosaic virus (CMV), Turnip yellow mosaic virus, Cauliflower mosaic virus (CaMV), Lettuce mosaic virus, or Plum pox virus. However, when the plants were preinfected with these viruses, TuMV was able to co-infect 12-4 plants preinfected with TRV, CaMV, and, particularly, CMV. Therefore, preinfection by another virus jeopardizes the amiRNA-mediated resistance to TuMV.
\end{abstract}

Most groups of eukaryotic organisms contain in their genomes microRNA (miRNA) genes, which are involved in the regulation of gene expression and epigenetic modifications (2). Genes coding for miRNAs are transcribed by the RNA polymerase II in long non-protein-coding primary-microRNA transcripts (primiRNAs), which are first processed to fold-back precursors (premiRNAs) and then matured to small double-stranded RNAs (3). The guide and passenger strands forming the RNA duplex are properly unfolded and only the mature miRNA (guide strand) is recruited by an Argonaute protein to form the active RNAinduced silencing complex (RISC). In plants, such as Arabidopsis thaliana L., Dicer-like1, an enzyme of the Dicer family with two RNase III domains, mediates cleavage of the pri- and pre-miRNAs. The miRNA loaded in RISC acts as a template to guide the silencing of complementary target mRNAs. In plants, miRNAs are usually highly complementary to their target, which typically triggers the cleavage of the targeted mRNA and subsequent degradation $(24,38)$.

Soon after the discovery of the basic steps of the miRNA processing pathway, it was shown that both animal and plant miRNA precursors could be engineered to express small RNAs whose sequences were unrelated to the corresponding mature miRNAs $(31,40)$. The utility of the artificial miRNA (amiRNA) technology was previously demonstrated in plants silencing genes with high specificity (1,36). Schwab et al. (36) designed and expressed a series of amiRNAs based on A. thaliana miRNA172a and miRNA319a backbones to target selected genes with notable phenotypic outcomes. The technology of amiRNAs was quickly adapted to produce virus-resistant plants. Niu et al. (30) generated A. thaliana transgenic lines expressing amiRNAs targeting two different 21-nucleotide (nt) regions in the genes coding for the

Corresponding author: J. A. Daròs; E-mail address: jadaros@ibmcp.upv.es

http://dx.doi.org/10.1094/PHYTO-09-12-0233-R

(c) 2013 The American Phytopathological Society
P69 and HC-Pro RNA-silencing suppressors of Turnip yellow mosaic virus (TYMV) (family Tymoviridae) and Turnip mosaic virus (TuMV) (family Potyviridae). Each amiRNA (amiR159-P69 and amiR159-HC-Pro) was produced from a 273-nt backbone based on the precursor of the endogenous amiR159a from A. thaliana and conferred specific resistance to the corresponding virus (30). Similar strategies based on the expression of one or co-expression of two or more amiRNAs conferred resistance to Cucumber mosaic virus (CMV) in tobacco (Nicotiana tabacum L.) (33), A. thaliana (10), and tomato (41); Potato virus $Y$ in tobacco (18); some negative-sense single-stranded RNA viruses in $N$. benthamiana Domin. plants (21); or Wheat streak mosaic virus in wheat (11).

Using two different $A$. thaliana transgenic lines expressing different levels of the amiR159-HC-Pro against TuMV (30), we have recently investigated the likelihood of emergence of virus variants able to break the amiRNA-mediated resistance (22). Although the $A$. thaliana transgenic line 12-4 expressing a high level of the amiR159-HC-Pro was remarkably resistant to TuMV infection, certain virus populations evolved by successive passaging in A. thaliana wild-type (WT) plants were able to break the resistance. All virus populations breaking resistance had fixed alleles with at least one mutation in the 21-nt sequence targeted by the amiRNA. Interestingly, virus evolved by successive passaging in the A. thaliana transgenic line 10-4 expressing a subinhibitory level of the amiR159-HC-Pro broke the resistance of the 12-4 line at a seven times higher rate (22). Characterization by ultradeep sequencing of some of the virus lineages that were able to break the resistance in the A. thaliana 12-4 transgenic line showed that, from the very first passage, the virus populations contained many alleles potentially able to break resistance, although at low frequencies. Some of these resistance-breaking alleles were predominant in the viral population once the resistance was broken in the A. thaliana 12-4 line, although they coexisted with the WT allele, now at low frequency (27). Not surprisingly, the frequencies of the potentially resistance-breaking 
alleles were more than one order of magnitude higher in the virus populations evolved in the A. thaliana transgenic line 10-4 expressing a subinhibitory amount of the amiR159-HC-Pro (27).

The goal of the present study was to analyze another potential cause of breaking the amiRNA-mediated antiviral resistance: namely, mixed viral infections. Plants use some of the RNAsilencing pathways as one of the main defense lines against invading viruses (9). Viruses respond with the expression of RNAsilencing suppressors to counteract the plant defense (23), and the expression of some viral RNA-silencing suppressors has been shown to interfere not only with the antiviral RNA-silencing pathways but also with the miRNA pathway (19), potentially affecting the amiRNA biogenesis. Here, we screened the fate of the amiR159-HC-Pro-mediated resistance to TuMV in mixed infections with viruses belonging to five different taxonomic groups. The virus accompanying TuMV was either co-inoculated or preinoculated in the A. thaliana 12-4 transgenic line. Our results illustrate how some viruses such as Tobacco rattle virus (TRV), Cauliflower mosaic virus (CaMV), and, notably, CMV help TuMV break the amiRNA-mediated resistance when they are already present in the plant at the moment of TuMV inoculation.

\section{MATERIALS AND METHODS}

Plant inoculations. To produce TuMV, Tobacco mosaic virus (TMV), TRV, CMV, TYMV, CaMV, Lettuce mosaic virus (LMV), and Plum pox virus (PPV) infectious extracts, adequate host plants for each virus were inoculated with an initial infectious material (see below). At $\approx 14$ days postinoculation (dpi), when infection symptoms were clearly observable, symptomatic tissues were harvested, aliquoted, and frozen and stored at $-80^{\circ} \mathrm{C}$. Aliquots of the different infected tissues were then ground with a mortar and pestle in the presence of liquid $\mathrm{N}_{2}$ and homogenized in 10 volumes of inoculation buffer $(50 \mathrm{mM}$ potassium phosphate, $\mathrm{pH}$ 8.0). The resulting crude extracts were used to mechanically inoculate batches of 3-week-old $A$. thaliana WT and transgenic (line 12-4, expressing a high level of amiR159-HC-Pro) plants. For this purpose, three aliquots of $5 \mu \mathrm{l}$ of $10 \%$ carborundum in inoculation buffer were applied onto three different fully expanded rosette leaves and a cotton swab, soaked with infectious extract (or 1:1 mix of extracts in co-inoculation experiments), was rubbed gently on the leaves' surface. In both the co-inoculation and preinoculation experiments, $4 \mathrm{~A}$. thaliana WT plants were inoculated with TuMV and TMV, TRV, TYMV, LMV, or PPV, and 20 A. thaliana WT plants were inoculated with TuMV and CMV or CaMV. In the case of the A. thaliana 12-4 plants, two batches of 20 plants were inoculated in two independent experiments (total of 40 plants) with TuMV and each one of the seven other viruses for both the co-inoculation and preinoculation. In preinoculation experiments, the other viruses were first inoculated and, a week later, three new fully expanded rosette leaves were inoculated with TuMV. Plants were kept in a growth chamber with cycles of $12 \mathrm{~h}$ of light at $25^{\circ} \mathrm{C}$ and $12 \mathrm{~h}$ of darkness at $23^{\circ} \mathrm{C}$.

To produce the initial TuMV infection, $N$. benthamiana plants were mechanically inoculated with a plasmid containing a TuMV cDNA (GenBank accession number AF530055.2 with a few point mutations) corresponding to isolate YC5 from calla lily (Zantedeschia sp.) (5) under the control of CaMV $35 \mathrm{~S}$ promoter (6). In the case of TMV, $N$. benthamiana plants were mechanically inoculated with $5^{\prime}$-capped transcripts produced in vitro from a plasmid containing a cDNA of the TMV-U1 strain (7). The initial infection of TRV was produced by agroinoculation of $N$. benthamiana plants with a 1:1 mix of two Agrobacterium tumefaciens transformed with plasmids containing the cDNAs corresponding to the RNA1 and RNA2 of this virus under the control of the CaMV 35S promoter (8). A mix of the three $5^{\prime}$-capped transcripts corresponding to the CMV-Fny strain were produced in vitro and mechanically inoculated in tobacco (34). The infection with
TYMV was started by mechanical inoculation of Arabidopsis thaliana with a plasmid containing a TYMV cDNA (32). Plasmid pCaMVW260 (35) was mechanically inoculated in Brassica rapa L. 'Just Right'. A plasmid with the cDNA of an LMV sequence variant consisting of a recombinant between LMV-E and LMVAF199 under the control of CaMV 35S promoter and Agrobacterium tumefaciens nos terminator $(20,29)$ was mechanically inoculated in N. benthamiana. Finally, the Rankovic strain of PPV under the control of $35 \mathrm{~S}$ promoter and nos terminator was agroinoculated in $N$. benthamiana (25).

Virus diagnosis. Fourteen days after the co-inoculation or after the second inoculation (preinoculation experiments), $100 \mathrm{mg}$ of tissue from various systemic leaves was harvested, frozen, and ground in a 2-ml Eppendorf tube with a 4-mm-diameter stainless steel ball using a Retsch MM300 mill for $1 \mathrm{~min}$ at $30 \mathrm{~s}^{-1}$. RNA or DNA was purified using silica gel spin columns eluting in $10 \mu \mathrm{l}$ (Zymo Research, Irving, CA). To reverse transcribe the viral RNAs, $1 \mu$ l of the RNA preparation was subjected to reverse transcription (RT) with $50 \mathrm{U}$ of $\mathrm{M}-\mathrm{MuLV}$ reverse transcriptase (Revertaid; Thermo-Scientific, Waltham, MA), $10 \mathrm{U}$ of RNase inhibitor (Thermo-Scientific), $1 \mathrm{mM}$ dNTPs, and 5 pmol of the appropriate primer depending on the diagnosed virus (Table 1) in a $10-\mu$ volume reaction with $50 \mathrm{mM}$ Tris- $\mathrm{HCl}(\mathrm{pH} 8.3), 50 \mathrm{mM}$ $\mathrm{KCl}, 4 \mathrm{mM} \mathrm{MgCl} 2$, and $10 \mathrm{mM}$ dithiothreitol for $45 \mathrm{~min}$ at $42^{\circ} \mathrm{C}$, $10 \mathrm{~min}$ at $50^{\circ} \mathrm{C}$, and $5 \mathrm{~min}$ at $60^{\circ} \mathrm{C}$. The mix of RNA and primer in $\mathrm{H}_{2} \mathrm{O}$ was denatured for $1.5 \mathrm{~min}$ at $98^{\circ} \mathrm{C}$ prior to addition of the remaining reagents. DNA or the cDNA product of the RT reaction $(1 \mu \mathrm{l})$ were subjected to polymerase chain reaction (PCR) amplification in 20- $\mu$ l-volume reactions with $1 \mathrm{U}$ of Thermus thermophilus DNA polymerase (Biotools, Madrid), $1 \mu \mathrm{M}$ each primer (Table 1), $0.2 \mathrm{mM}$ dNTPs, $75 \mathrm{mM}$ Tris- $\mathrm{HCl}(\mathrm{pH} 9.0), 2 \mathrm{mM}$ $\mathrm{MgCl}_{2}, 50 \mathrm{mM} \mathrm{KCl}$, and $20 \mathrm{mM}\left(\mathrm{NH}_{4}\right)_{2} \mathrm{SO}_{4}$. Reactions were incubated for $2 \mathrm{~min}$ at $94^{\circ} \mathrm{C}$; followed by 30 cycles of $40 \mathrm{~s}$ at $94^{\circ} \mathrm{C}, 30 \mathrm{~s}$ at $55^{\circ} \mathrm{C}$, and $3 \mathrm{~min}$ at $72^{\circ} \mathrm{C}$; with a final extension of $10 \mathrm{~min}$ at $72^{\circ} \mathrm{C}$. PCR products were analyzed by electrophoresis in $1 \%$ agarose gels in Tris-acetate EDTA buffer (40 mM Tris, 20 $\mathrm{mM}$ sodium acetate, and $1 \mathrm{mM}$ EDTA, $\mathrm{pH}$ 7.2). Gels were stained with ethidium bromide.

AmiR159-HC-Pro quantification. Total RNA was extracted from three different fully expanded rosette leaves of three independent 4-week-old amiR159-HC-Pro-expressing Arabidopsis thaliana plants (line 12-4) noninoculated or preinfected by TMV, TRV, CMV, TYMV, CaMV, LMV, or PPV using the Trizol reagent (Invitrogen, Life Technologies, Paisley, UK). The quantification of amiR159-HC-Pro in the RNA preparations was performed by RT-quantitative PCR as described before (22).

Statistical analyses. In single-infection experiments, Fisher's exact probability tests for two-by-two contingency tables (37) were used to evaluate the effect of A. thaliana genotype (WT versus 12-4) on the infectivity of each virus. In multiple-inoculation experiments, Fisher's exact probability tests for two-bythree contingency tables (12) were used to evaluate the effect of A. thaliana genotype or inoculation scheme (pre- versus coinoculation) in TuMV infectivity in the presence of the different companion viruses. The same test was also used to discard block effects in the case of the A. thaliana 12-4 plants. Infectivity data were also analyzed in a different way. An infection interference index was computed between pairs of co-infecting viruses as

$$
S_{\text {TuMV }, \mathrm{y}}=p_{\text {TuMV }, \mathrm{y}}-p_{\mathrm{TuMv}} p_{\mathrm{y}}
$$

where $p_{\text {TuMv,y }}$ was the observed frequency of plants simultaneously infected by TuMV and companion virus $y$, and $p_{\text {TuMV }}$ and $p_{\mathrm{y}}$ were the observed frequencies of infection for TuMV and virus $y$ in single-inoculation experiments (Table 2). If the infectivity of TuMV is independent of the presence of the companion virus $y$, then observed and expected $\left(p_{\text {TuMv }} p_{\mathrm{y}}\right)$ frequencies will be 
equal and, thus, $S_{\mathrm{TuMV}, \mathrm{y}}=0$. Values of $S_{\mathrm{TuMV}, \mathrm{y}}$ significantly deviating from this null expectation imply synergistic (positive) or antagonistic (negative) interference during infection. To evaluate the statistical significance of $S_{\mathrm{TuMV}, \mathrm{y}}$ estimates, we proceeded as follows. First, the standard error of every $p$ in equation 1 was calculated as

$$
\sigma_{p}=\sqrt{p(1-p) / n}
$$

(39), where $n$ is the number of plants inoculated for a given trial. Second, we applied Fisher's $\Delta$ method for error propagation to equation1 to obtain the standard error of $S_{\mathrm{TuMV}, \mathrm{y}}$,

$$
\sigma_{S_{\mathrm{TuMV}, y}}=\sqrt{\sigma_{p_{\mathrm{TuMV}, y}}^{2}+p_{y}^{2} \sigma_{p_{\mathrm{TuMV}}}^{2}+p_{\mathrm{TuMV}}^{2} \sigma_{p_{y}}^{2}}
$$

Finally, statistical significance of $S_{\text {TuMv,y }}$ was evaluated using the standard normal distribution. Other statistical tests were introduced when necessary. All analyses were performed using IBM SPSS (v. 21; Armonk, NY).

\section{RESULTS}

Co-inoculation of the $A$. thaliana transgenic line 12-4 expressing the amiR159-HC-Pro with TuMV and a series of other viruses. The $A$. thaliana transgenic line 12-4 that expresses a high level of the antiviral amiR159-HC-Pro, which targets $21 \mathrm{nt}$ in the TuMV cistron coding for HC-Pro (Fig. 1), is highly resistant to TuMV infection $(22,30)$. To investigate the outcome of this resistance when the plants are challenged by two different viruses in mixed infections, plants were co-inoculated with infectious extracts of TuMV and other viruses belonging to the same or different taxonomic groups. We chose TMV (genus Tobamovirus) and TRV (genus Tobravirus), both belonging to the family Virgaviridae; CMV (family Bromoviridae); TYMV (family Tymoviridae); and CaMV (family Caulimoviridae) as representatives from phylogenetically unrelated families. We also chose two viruses belonging, like TuMV, to the genus Potyvirus (family Potyviridae): LMV and PPV. All these viruses and, particularly, the sequence variants used in this study were previously described to be highly infectious in the ecotype Col-0 of $A$. thaliana. Nonetheless, we checked their infectivity in our particular experimental conditions.
Using plasmids containing the DNA (CaMV) or the cDNAs (all the other) of the different viruses, we first infected a typical host plant for each virus. As soon as the plants showed symptoms, the symptomatic tissues were harvested and frozen. Aliquots of these tissues were used to produce the different infectious extracts that were employed to mechanically inoculate WT and amiR159-HCPro-expressing (line 12-4) A. thaliana plants. At 14 dpi, infection by the different viruses was diagnosed by PCR (CaMV) or RTPCR (all the other viruses) and subsequent electrophoretic analysis of the amplification products. All extracts were highly infectious in WT A. thaliana plants (Table 2). All extracts, except TuMV $(P<0.001)$ were also highly infectious in $12-4 A$. thaliana plants (Table 2).

Next, we sought to evaluate whether co-inoculation of plants with other viruses affected the infectivity of TuMV, in particular in the highly resistant 12-4 plants. To do so, we co-inoculated WT and 12-4 A. thaliana plants with TuMV and each one of the other viruses. At 14 dpi, systemic tissues were harvested, total RNA or DNA purified, and the virus infections diagnosed. Co-inoculation of the transgenic 12-4 plants was carried out independently twice with two batches of 20 plants. Results of both replicates of the experiment were homogenous ( $P=1$ in all cases); therefore, we combined the results to calculate the ratios of infected plants. The ratio of plants that were co-infected with both viruses or singly infected with the other virus or with TuMV is shown in Figure 2A. In the co-inoculations with TMV, TRV, and LMV, all WT plants were co-infected with both viruses, whereas most 12-4

TABLE 2. Infectivity of the different infectious extracts in plants of the Arabidopsis thaliana wild type (WT) and the transgenic line 12-4 expressing high levels of the antiviral amiR159-HC-Pro ${ }^{\mathrm{a}}$

\begin{tabular}{lcc}
\hline Virus & WT & Line $12-4$ \\
\hline Turnip mosaic virus & $4 / 4$ & $0 / 20$ \\
Tobacco mosaic virus & $4 / 4$ & $20 / 20$ \\
Tobacco rattle virus & $3 / 4$ & $14 / 18$ \\
Cucumber mosaic virus & $18 / 20$ & $18 / 20$ \\
Turnip yellow mosaic virus & $4 / 4$ & $20 / 20$ \\
Cauliflower mosaic virus & $19 / 20$ & $18 / 20$ \\
Lettuce mosaic virus & $4 / 4$ & $20 / 20$ \\
Plum pox virus & $4 / 4$ & $15 / 18$ \\
\hline
\end{tabular}

a Number of infected plants (14 days postinoculation) over the number of inoculated plants.

TABLE 1. Primers used in virus diagnosis by reverse-transcription polymerase chain reaction (RT-PCR) and PCR

\begin{tabular}{lll}
\hline Virus & Reaction & Deoxyoligonucleotide sequence (from $5^{\prime}$ to $\left.3^{\prime}\right)^{\mathrm{a}}$ \\
\hline Turnip mosaic virus & RT & TGTCCCTTGCATCCTATCAAATGTTAAG \\
& PCR & GCAGGTGAAACGCTTGATGCAGGTTTG (F) \\
Tobacco mosaic virus & RT & CAACCCCTGAACGCCCAGTAAGTTATG (R) \\
& PCR & CGCTTTATTACGTGCCTGCGGATGT \\
Tobacco rattle virus & RT & GTGGATGAGTTCGTGGAAGATGTC (F) \\
& PCR & CTAATGGCATCAGTGAATATGGTATCACC \\
Cucumber mosaic virus & RT & TAGGGATTAGGACGTATCGGACCTC (F) \\
& PCR & GTCCTGCTGACTTGATGGACGATTC (R) \\
Turnip yellow mosaic virus & RT & TCAGACTGGGAGCACTCCAGACG \\
& PCR & ATGGACAAATCTGAATCAACC (F) \\
Cauliflower mosaic virus & & TCAGACTGGGAGCACTCCAGACG (R) \\
Lettuce mosaic virus & PCR & GGTGGAAGTGTCCGTGATGAGCGG \\
& CGACAAAGACTCGCCCCAAG (F) \\
Plum pox virus & RT & GGTGGAAGTGTCCGTGATGAGCGG (R) \\
& PCR & ATCAACAAAGTTGATGG (F) \\
& RT & GAAGGTATT TGATCTCCTG (R) \\
& PCR & CACACACTAGCAACCTTCGAGG \\
\hline
\end{tabular}

$\mathrm{a}(\mathrm{F})$ and $(\mathrm{R})$ indicate forward and reverse, respectively. 
plants were singly infected by TMV, TRV, or LMV, and these differences in infectivity between plant genotypes were highly significant $(P<0.001$ in all three cases). The results with CMV were similar but, in this case, only $70 \%$ of the WT plants were coinfected and $67 \%$ of the $12-4$ plants were infected by only CMV (test of equal infectivity between plant genotypes: $P<0.001$ ). In the case of CaMV, results were more complex because $75 \%$ of the WT plants were doubly infected and the remaining $25 \%$ were infected just with TuMV, whereas $20 \%$ of the $12-4$ plants were infected by only CaMV and the other $80 \%$ not infected at all (test of equal infectivity between plant genotypes: $P<0.001)$. In the co-inoculation with TuMV and TYMV, all WT and 12-4 plants were singly infected by TYMV. Finally, in the co-inoculation with TuMV and PPV, TuMV singly infected all WT plants whereas most 12-4 plants were infected only by PPV. In all cases, not a single amiR159-HC-Pro-expressing 12-4 plant was infected by TuMV (Fig. 2A).

The infectivity indexes $S_{\text {TuMv,y }}$ estimated for TuMV and each one of the seven companion viruses obtained for the co-infection experiments are shown in Table 3. Interestingly, TYMV and PPV showed significant antagonism with TuMV in WT A. thaliana plants $\left(S_{\text {TuMV ,y }}<0\right)$. Because TuMV was not found in any of the co-inoculated 12-4 plants, $S_{\mathrm{TuMV}, \mathrm{y}}=0$ in all cases (Table 3 ).

In conclusion, although there appear to be interactions and interference between some of the viruses and TuMV when coinoculated, we observed no impact of the co-inoculation with any one of the seven companion viruses on the ability of TuMV to break the amiR159-HC-Pro-mediated resistance of 12-4 plants.

Inoculation with TuMV of amiR159-HC-Pro-expressing A. thaliana plants already infected with another virus. To further investigate the resistance of the A. thaliana 12-4 line to TuMV in mixed infections, we performed experiments in which plants were preinoculated with the other viruses and then inoculated with TuMV at $7 \mathrm{dpi}$, once the infection by these other viruses was established. Fourteen days after the second inoculation, DNA or RNA was purified from systemic tissues and the infection by the different viruses diagnosed by PCR or RT-PCR (Fig. 2B). Those plants that were diagnosed as noninfected by the first virus after the preinoculation were discarded from the analysis. Again, preinoculation of the transgenic 12-4 plants was carried out independently twice with two batches of 20 plants. Results of both replicates of the experiment were also homogenous $(P \geq 0.229$ in all cases) and the counts combined to calculate ratios of infected plants.

The new experimental setup did not alter the results in the cases of TMV and LMV: all WT plants were co-infected with both viruses whereas all of the 12-4 plants were singly infected by TMV or LMV (Fig. 2B). Surprisingly, the preinoculation approach in the case of TYMV gave the same result as TMV and LMV, and was radically different from what was observed in the co-inoculation experiment: all WT plants were infected by both

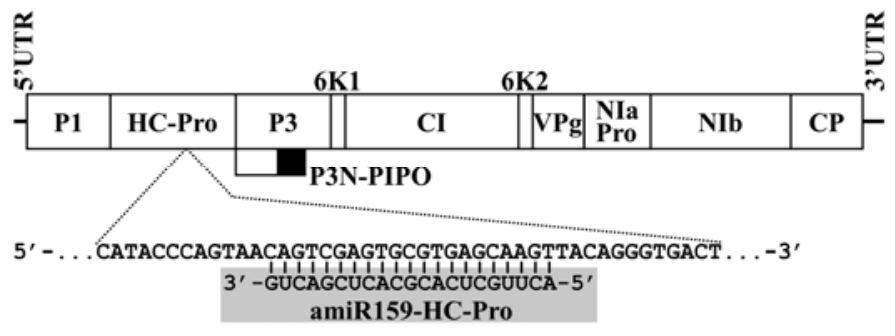

Fig. 1. Schematic representation of the Turnip mosaic virus (TuMV) genome with a fragment of the sequence in the HC-Pro cistron containing the 21nucleotide complementary target of the amiR159-HC-Pro (boxed on gray background) expressed in the Arabidopsis thaliana transgenic line 12-4. Boxes P1, HC-Pro, P3, P3N-PIPO, 6K1, CI, 6K2, VPg, NIaPro, NIb, and CP represent the cistrons coding for the different TuMV gene products. Lines $5^{\prime} \mathrm{UTR}$ and $3^{\prime} \mathrm{UTR}$ represent the $5^{\prime}$ and $3^{\prime}$ untranslated regions, respectively. viruses whereas all 12-4 plants were infected only by TYMV (Fig. 2B). Identical results were obtained for plants preinfected with PPV: all WT plants were co-infected by both viruses whereas all 12-4 plants were singly infected by PPV (Fig. 2B). This lack of effect of the inoculation method (co- or preinoculation) on TuMV infectivity was consistent with the nonsignificant result of Fisher's exact probability tests run for each of these viruses $(P=1$ in all cases). However, preinfection of the 12-4 plants with TRV, $\mathrm{CMV}$, and CaMV significantly helped TuMV break the resistance provided by the amiRNA in a variable extent. In the case of CaMV, all the WT plants were doubly infected by CaMV and TuMV, whereas $\approx 36 \%$ of the infected $12-4$ plants were doubly infected (Fig. 2B). Nonetheless, the effect of the inoculation method was not significant for $\mathrm{CaMV}$ in either plant genotype $(P=0.182$ for WT and $P=0.076$ for $12-4)$. Similarly, in the case of TRV, all the WT plants were doubly infected by TRV and TuMV, whereas $\approx 29 \%$ of the $12-4$ plants were co-infected by TRV and TuMV (Fig. 2B), with the difference of the inoculation method significant in the 12-4 plants $(P=0.01)$ but not in the WT plants $(P=1)$. Finally, the most remarkable results were obtained for CMV: all WT and $\approx 94 \%$ of the $12-4$ plants were doubly infected by both CMV and TuMV (Fig. 2B). The inoculation method has no effect for the WT plants $(P=1)$ whereas it has a highly significant effect on the $12-4$ plants $(P<0.001)$.
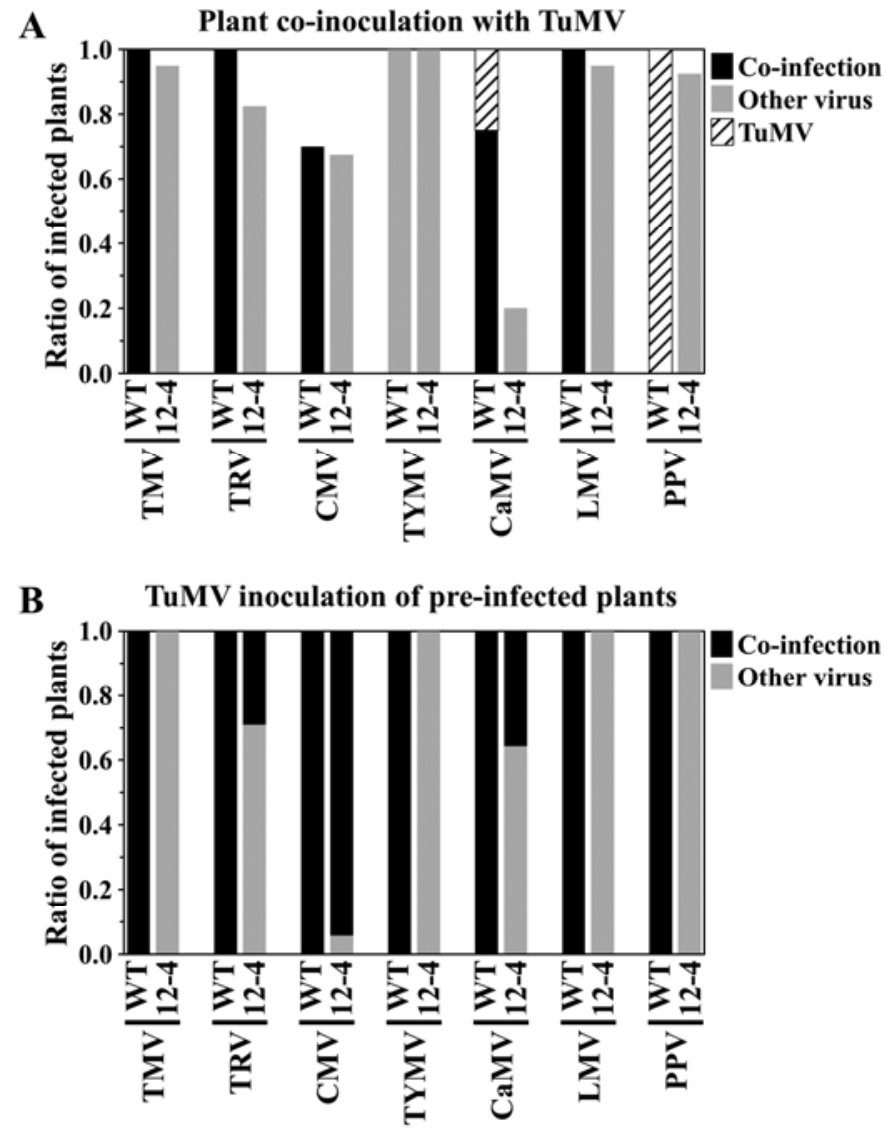

Fig. 2. Infectivity of Turnip mosaic virus (TuMV) in mixed inoculations with Tobacco mosaic virus (TMV), Tobacco rattle virus (TRV), Cucumber mosaic virus (CMV), Turnip yellow mosaic virus (TYMV), Cauliflower mosaic virus (CaMV), Lettuce mosaic virus (LMV), and Plum pox virus (PPV) in wild-type (WT) and amiR159-HC-Pro-expressing 12-4 Arabidopsis thaliana plants. A, Plants were co-inoculated and the viruses diagnosed 14 days post inoculation (dpi). B, Plants were preinfected with the other virus and inoculated with TuMV at 7 dpi. Plants were diagnosed 14 days after the second inoculation. Black, gray, and striped columns indicate plants co-infected, singly infected by the other virus, or singly infected by TuMV, respectively. Note that, in B, because only plants preinfected by the other virus were inoculated with TuMV, no TuMV singly infected plants (striped column) could exist. 
The infection interference indexes $S_{\text {TuMv,y }}$ computed for TuMV and each one of the seven companion viruses in the preinoculation experiments are shown in Table 3. In agreement with the results described in the previous paragraph, the outcome of TuMV infection was independent of whether the companion virus was present or absent (no significant deviations from the independent action hypothesis) in WT plants. By contrast, TRV, CMV, and CaMV show highly significant positive synergy with TuMV (Table 3) when preinoculated in the 12-4 plants.

In conclusion, we observed that preinfection with TRV, CaMV, and, particularly, CMV had a positive effect on the ability of TuMV to infect and accumulate in the 12-4 resistant plants.

Effect of virus infection on amiR159-HC-Pro concentration. After observing that preinfection with some viruses helps TuMV break the resistance mediated by the amiR159-HC-Pro in the 12-4 A. thaliana plants, we measured the amiR159-HC-Pro concentration, in triplicate, in noninoculated plants (control) and in plants preinfected by each one of the seven companion viruses. The tissues assayed for amiR159-HC-Pro concentration were equivalent to those inoculated with TuMV in the preinoculation experiments. The amiR159-HC-Pro concentration increased in all infected plants compared with the noninoculated control (Fig. 3). The lowest accumulation corresponded to CaMV-infected plants (1.37 times more than the control) and the largest to plants infected with TYMV (2.5-fold increase relative to the control). A Kruskal-Wallis test found significant overall differences among experiments $(H=15.760,7 \mathrm{df}, P=0.027)$ and a post hoc Tukey's honestly significant difference test identified two homogenous but overlapping groups (Fig. 3, a and b). Significant pairwise differences were only found between the negative control and TMV and TYMV. Interestingly, no obvious association exists between the level of amiR159-HC-Pro accumulation in plants infected with the other viruses and whether TuMV was able to accumulate.

\section{DISCUSSION}

The amiRNA technology has provided some promising results toward the aim of obtaining a new generation of crop plants resistant to viruses $(10,11,18,21,30,33,41)$. In addition to a strong virus resistance achieved in some transgenic lines expressing high levels of one or more amiRNAs, the technology offers the advantages of little risk of off-target effects and negligible chance of recombination with other invading viral genomes, due to the small size of the mature amiRNAs. However, as advanced when the antiviral properties of the technology in plants were first demonstrated (13), viruses and, particularly, RNA viruses, thanks to their amazing evolutionary potential, are able to cope with the resistance.
The A. thaliana transgenic line 12-4 that expresses a high level of the amiR159-HC-Pro (Fig. 1) is highly resistant to TuMV infection when the plants are inoculated with a virus population amplified by simply infecting a susceptible host plant with a particular TuMV cDNA containing the 21-nt target of the amiRNA $(22,30)$. However, when the viral population is allowed to evolve by successive passaging from plant to plant, the amiRNA-mediated resistance is finally broken (22). Nonetheless, the performance of this resistance trait was remarkable, because 25 independent lineages of TuMV took a median of 14 evolutionary passages from WT to WT $A$. thaliana to finally break resistance (22). Therefore, it is worth exploring the potentiality and limitations of this technology as an antiviral strategy to manage it properly in a future and more biotechnological agriculture. For example, Duan et al. (10) showed that the antiviral efficacy of amiRNAs depends on the local structures of the target RNAs. Also, Kung et al. (21) showed that the levels of virus resistance are positively correlated with the amiRNA expression levels and that expression of more than one amiRNA has an additive effect. We have previously shown that plants expressing subinhibitory amounts of the antiviral amiRNA jeopardize the resistance, because the virus population infecting these plants contains a higher frequency of alleles with mutations in the amiRNA target potentially able to break resistance $(22,27)$.

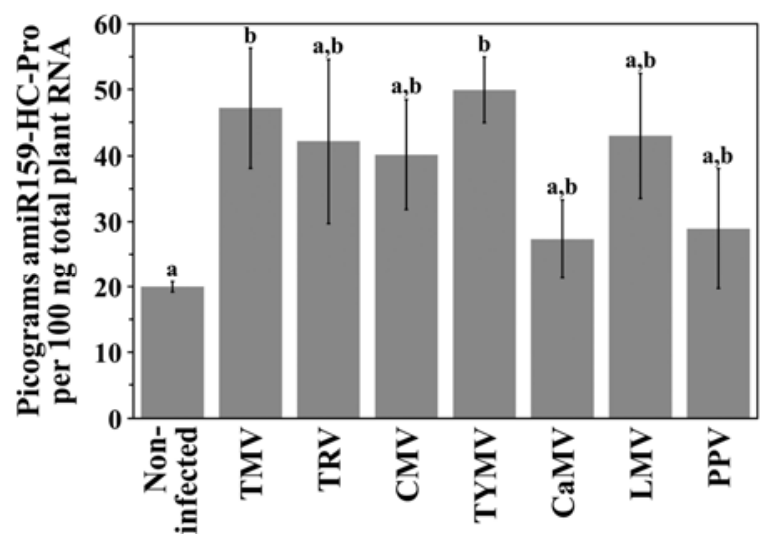

Fig. 3. Accumulation of the amiR159-HC-Pro in Arabidopsis thaliana plants (line 12-4) noninfected and infected by Tobacco mosaic virus (TMV), Tobacco rattle virus (TRV), Cucumber mosaic virus (CMV), Turnip yellow mosaic virus (TYMV), Cauliflower mosaic virus (CaMV), Lettuce mosaic virus (LMV), and Plum pox virus (PPV). Columns represent the average amount of artificial microRNA (amiRNA) (picograms per $100 \mathrm{ng}$ of total plant RNA) in fully expanded rosette leaves of three plants. Error bars indicate $\pm 1 \sigma$. Letters a and $b$ indicate homogeneous groups according to a Tukey's honestly significant difference post hoc test.

TABLE 3. Infection interference index $\left(S_{\text {TuMV }, y} \pm 1 \sigma_{s}\right)$ computed for Turnip mosaic virus (TuMV) and each of the seven companion viruses $(y)$ in both Arabidopsis thaliana genotypes and using both inoculation schemes ${ }^{\mathrm{a}}$

\begin{tabular}{lll}
\hline Companion virus & A. thaliana wild type & A. thaliana $12-4$ \\
\hline Co-inoculation experiments & 0 & 0 \\
Tobacco mosaic virus & $0.250 \pm 0.217(P=0.124)$ & 0 \\
Tobacco rattle virus & $0.100 \pm 0.067(P=0.068)$ & 0 \\
Cucumber mosaic virus & -1 & 0 \\
Turnip yellow mosaic virus & $-0.2 \pm 0.146(P=0.146)$ & 0 \\
Cauliflower mosaic virus & 0 & 0 \\
Lettuce mosaic virus & -1 & 0 \\
Plum pox virus & 0 & 0 \\
Preinoculation experiments & 0 & $0.290 \pm 0.082(P<0.001)$ \\
Tobacco mosaic virus & $0.047 \pm 0.118(P=0.344)$ & $0.943 \pm 0.039(P<0.001)$ \\
Tobacco rattle virus & 0 & 0 \\
Cucumber mosaic virus & $0 \pm 0.097(P=0.500)$ & $0.357 \pm 0.091(P<0.001)$ \\
Turnip yellow mosaic virus & 0 & 0 \\
Cauliflower mosaic virus & 0 & 0 \\
Lettuce mosaic virus & 0.000 \\
Plum pox virus &
\end{tabular}

a The significance of the null hypothesis of no interference $\left(S_{\mathrm{TuMV}, \mathrm{y}}=0\right)$ was evaluated using the standard normal distribution $z$ test (provided in parenthesis). 
In this study, we analyzed the fate of the amiR159-HC-Promediated resistance to TuMV of the A. thaliana transgenic line 12-4 in mixed viral infections, a frequent situation in nature. For this purpose, we screened the ability of TuMV to infect the $A$. thaliana transgenic line 12-4 when co-inoculated with another virus and when inoculated in plants that were already infected with another virus. In these experiments, we used a total of seven different companion viruses: four (+)-strand RNA viruses (TMV, TRV, CMV, and TYMV) and one DNA virus (CaMV) belonging to four different families apart from the Potyviridae, and two RNA viruses (LMV and PPV) belonging to the same genus (Potyvirus) and family (Potyviridae) as TuMV. All seven viruses assayed in this study, or at least the sequence variants used here, were highly infectious in the ecotype Col-0 of A. thaliana, and this high infectivity was not affected by the expression of the antiTuMV amiR159-HC-Pro (Table 2).

The amiR159-HC-Pro protected the A. thaliana plants from TuMV infection in the co-inoculation experiments. Not a single plant belonging to the 12-4 line was infected by TuMV (Fig. 2A). In contrast, most of the WT plants were co-infected by TuMV and the companion virus. A notable exception to this observation was co-inoculation of WT plants with TYMV: not a single WT plant was infected by TuMV, suggesting an antagonistic effect of TYMV on TuMV infection $\left(S_{\text {TuMV,TYMV }}<0\right)$ (Table 3$)$. Another exception was PPV: all WT plants were singly infected by TuMV and all 12-4 plants were singly infected by PPV. In this case, the antagonistic effect is of TuMV on PPV infection of WT plants (Table 3). An antagonistic effect is also suggested by the results of the co-inoculation with CaMV. TuMV infected all the WT plants and most were co-infected by CaMV but TuMV also singly infected some plants. In this case, the antagonistic effect is of TuMV on CaMV infection, although the effect was not strong enough as to reach statistical significance (Table 3). Interestingly, although TuMV did not infect a single A. thaliana 12-4 plant when co-inoculated with $\mathrm{CaMV}$, this second virus was only able to infect a few (20\%) of these plants (Fig. 2A). This result indicates that the antagonistic effect of TuMV on CaMV infection might occur even though TuMV itself is not able to establish a systemic infection due to the presence of the anti-TuMV amiRNA. If this interpretation is correct, the amiRNA blockage to the TuMV infection would occur in a step of TuMV infection in which this virus would have already interfered with the CaMV infection.

The amiRNA-mediated protection of the A. thaliana plants against TuMV in the preinoculation experiments was also notable but, in this case, some of the viruses definitively helped TuMV break the resistance (Fig. 2B). Preinfection with TMV, TYMV, LMV, and PPV did not have any effect on TuMV's ability to break resistance. Not a single 12-4 plant was infected by TuMV (inoculated at 7 days after the first virus) whereas all of the WT plants were co-infected by TuMV and the second virus. It is interesting to note that, although TYMV blocked TuMV infection and TuMV blocked PPV infection of the WT plants in the coinoculation experiments, this effect was not observed in the preinoculation experiments, indicating that the antagonistic effect extends exclusively to the initial steps of infection. TRV and CaMV helped TuMV break the resistance provided by the amiRNA in the preinoculation experiments: some of the 12-4 plants were co-infected by TRV and TuMV, and CaMV and TuMV (Fig. 2B). The most remarkable case, however, was CMV. Most 12-4 plants preinfected with CMV were also co-infected with TuMV (Fig. 2B).

Our experiments do not allow us to dissect why TRV and CaMV, and particularly CMV, help TuMV break the amiRNAmediated resistance; however, we can speculate that the RNAsilencing suppressors expressed by these viruses somehow interfere with the expression, maturation, stability, or activity of the amiR159-HC-Pro, clearing the way for TuMV infection. Quanti- fication of the amiR159-HC-Pro in A. thaliana plants preinfected with the seven viruses and in noninoculated controls showed that, in all cases, infection with the companion viruses increases the concentration of the amiRNA in leaf tissues at 7 dpi (Fig. 3; the increase with respect to the noninoculated control was not statistically significant in CaMV and PPV). This result indicates that the TuMV resistance breakdown induced by CMV, CaMV, and TRV is not caused by a reduction in the amiRNA concentration but, possibly, by affecting the functionality of the amiRNA. The CMV 2b protein has been shown to be a suppressor of RNA silencing (4). Despite being a small protein, it interacts with many factors and pathways in the infected host (15). However, two activities are considered crucial to interfere with the plant antiviral RNA-silencing pathways: the siRNA binding activity (14) and the Argonaute1 binding activity that inhibits slicing by RISC (42). Interestingly, CMV $2 b$ has also been shown to bind miRNA in vivo (17). In the case of TRV, the RNAsilencing suppressor activity has been associated with the $16-\mathrm{kDa}$ protein (28). This is considered a weak suppressor not affecting the endogenous miRNA pathway; however, in our preinoculation experiment, preinfection with TRV helped TuMV break the amiR159-HC-Pro-mediated resistance in $29 \%$ of the plants, suggesting some kind of interference with the amiRNA pathway. Finally, the P6 protein is the viral RNA-silencing suppressor of CaMV (26). This protein interacts with the double-stranded RNAbinding protein DRB4 that facilitates the activity of the major plant antiviral silencing factor Dicer-like4 (16). Again, our results suggest some kind of interference of the CaMV infection with the amiRNA activity.

In our study, we included two potyviruses, LMV and PPV. None of them helped TuMV break the resistance provided by the amiR159-HC-Pro in either the co-inoculation or preinoculation assay, indicating that HC-Pro (or the tandem P1/HC-Pro), the strong RNA-silencing suppressor of potyviruses, does not interfere with amiRNA-mediated resistance. We did not anticipate this result because it was previously described that tandem expression of TuMV P1 and HC-Pro in A. thaliana plants interfered with the endogenous miRNA pathway producing developmental alterations (19). In any case, this result may explain why the amiR159HC-Pro is so effective at protecting the A. thaliana plants against a potyvirus like TuMV. Although, in the initial steps of infection, the virus was able to deploy its machinery of RNA-silencing suppression, this would not affect the activity of the amiRNA. If this interpretation is correct, it would support, at least in the case of potyviruses, another of the advantages that has been associated with the technology of the antiviral amiRNAs. In contrast to other host RNA-silencing pathways, like that of the small-interfering RNAs, host plants do not normally employ the miRNA pathway in antiviral roles and, therefore, plant viruses have not evolved counter-measures to avoid the surveillance of the miRNA pathways.

\section{ACKNOWLEDGMENTS}

This research was supported by the CSIC grant 2010 TW0015 (to J. A. Daròs and S. F. Elena), the Generalitat Valenciana grant PROMETEO/ 2010/019 (to S. F. Elena and J. A. Daròs), and the Spanish Ministerio de Economía y Competitividad grants BIO2011-26741 (to J. A. Daròs) and BFU2012-30805 (to S. F. Elena). F. Martínez was supported by a predoctoral fellowship from the Universidad Politécnica de Valencia.

\section{LITERATURE CITED}

1. Alvarez, J. P., Pekker, I., Goldshmidt, A., Blum, E., Amsellem, Z., and Eshed, Y. 2006. Endogenous and synthetic microRNAs stimulate simultaneous, efficient, and localized regulation of multiple targets in diverse species. Plant Cell 18:1134-1151.

2. Ambros, V. 2008. The evolution of our thinking about microRNAs. Nat. Med. 14:1036-1040 
3. Bartel, D. P. 2004. MicroRNAs: genomics, biogenesis, mechanism, and function. Cell 116:281-297.

4. Brigneti, G., Voinnet, O., Li, W. X., Ji, L. H., Ding, S. W., and Baulcombe, D. C. 1998. Viral pathogenicity determinants are suppressors of transgene silencing in Nicotiana benthamiana. EMBO J. 17:67396746.

5. Chen, C. C., Chao, C. H., Yeh, S. D., Tsai, H. T., and Chang, C. A. 2003. Identification of Turnip mosaic virus isolates causing yellow stripe and spot on calla lily. Plant Dis. 87:901-905.

6. Chen, C. C., Chen, T. C., Raja, J. A., Chang, C. A., Chen, L. W., Lin, S. S., and Yeh, S. D. 2007. Effectiveness and stability of heterologous proteins expressed in plants by Turnip mosaic virus vector at five different insertion sites. Virus Res. 130:210-227.

7. Chen, J., Watanabe, Y., Sako, N., Ohshima, K., and Okada, Y. 1996. Complete nucleotide sequence and synthesis of infectious in vitro transcripts from a full-length cDNA clone of a rakkyo strain of tobacco mosaic virus. Arch. Virol. 141:885-900.

8. Dinesh-Kumar, S. P., Anandalakshmi, R., Marathe, R., Schiff, M., and Liu, Y. 2003. Virus-induced gene silencing. Methods Mol. Biol. 236:287294.

9. Ding, S. W., and Voinnet, O. 2007. Antiviral immunity directed by small RNAs. Cell 130:413-426.

10. Duan, C. G., Wang, C. H., Fang, R. X., and Guo, H. S. 2008. Artificial microRNAs highly accessible to targets confer efficient virus resistance in plants. J. Virol. 82:11084-11095

11. Fahim, M., Millar, A. A., Wood, C. C., and Larkin, P. J. 2012. Resistance to Wheat streak mosaic virus generated by expression of an artificial polycistronic microRNA in wheat. Plant Biotechnol. J. 10:150-163.

12. Freeman, G. H., and Halton, J. H. 1951. Note on an exact treatment of contingency, goodness of fit and other problems of significance. Biometrika 38:141-149.

13. García, J. A., and Simón-Mateo, C. 2006. A micropunch against plant viruses. Nat. Biotechnol. 24:1358-1359.

14. González, I., Martínez, L., Rakitina, D. V., Lewsey, M. G., Atencio, F. A., Llave, C., Kalinina, N. O., Carr, J. P., Palukaitis, P., and Canto, T. 2010. Cucumber mosaic virus $2 \mathrm{~b}$ protein subcellular targets and interactions: their significance to RNA silencing suppressor activity. Mol. PlantMicrobe Interact. 23:294-303.

15. González, I., Rakitina, D., Semashko, M., Taliansky, M., Praveen, S., Palukaitis, P., Carr, J. P., Kalinina, N., and Canto, T. 2012. RNA binding is more critical to the suppression of silencing function of Cucumber mosaic virus $2 \mathrm{~b}$ protein than nuclear localization. RNA 18:771-782.

16. Haas, G., Azevedo, J., Moissiard, G., Geldreich, A., Himber, C., Bureau, M., Fukuhara, T., Keller, M., and Voinnet, O. 2008. Nuclear import of CaMV P6 is required for infection and suppression of the RNA silencing factor DRB4. EMBO J. 27:2102-2112.

17. Hamera, S., Song, X., Su, L., Chen, X., and Fang, R. 2012. Cucumber mosaic virus suppressor $2 \mathrm{~b}$ binds to AGO4-related small RNAs and impairs AGO4 activities. Plant J. 69:104-115.

18. Jiang, F., Song, Y. Z., Han, Q. J., Zhu, C. X., and Wen, F. J. 2011. The choice of target site is crucial in artificial miRNA-mediated virus resistance in transgenic Nicotiana tabacum. Physiol. Mol. Plant Pathol. 76:28 .

19. Kasschau, K. D., Xie, Z., Allen, E., Llave, C., Chapman, E. J., Krizan, K. A., and Carrington, J. C. 2003. P1/HC-Pro, a viral suppressor of RNA silencing, interferes with Arabidopsis development and miRNA function. Dev. Cell 4:205-217.

20. Krause-Sakate, R., Redondo, E., Richard-Forget, F., Jadao, A. S., Houvenaghel, M. C., German-Retana, S., Pavan, M. A., Candresse, T., Zerbini, F. M., and Le Gall, O. 2005. Molecular mapping of the viral determinants of systemic wilting induced by a Lettuce mosaic virus (LMV) isolate in some lettuce cultivars. Virus Res. 109:175-180.

21. Kung, Y. J., Lin, S. S., Huang, Y. L., Chen, T. C., Harish, S. S., Chua, N. H., and Yeh, S. D. 2012. Multiple artificial microRNAs targeting conserved motifs of the replicase gene confer robust transgenic resistance to negative-sense single-stranded RNA plant virus. Mol. Plant Pathol. 13:303-317.
22. Lafforgue, G., Martínez, F., Sardanyés, J., de la Iglesia, F., Niu, Q. W. Lin, S. S., Solé, R. V., Chua, N. H., Daròs, J. A., and Elena, S. F. 2011. Tempo and mode of plant RNA virus escape from RNA interferencemediated resistance. J. Virol. 85:9686-9695.

23. Li, F., and Ding, S. W. 2006. Virus counterdefense: diverse strategies for evading the RNA-silencing immunity. Annu. Rev. Microbiol. 60:503-531.

24. Llave, C., Kasschau, K. D., Rector, M. A., and Carrington, J. C. 2002. Endogenous and silencing-associated small RNAs in plants. Plant Cell $14: 1605-1619$

25. López-Moya, J. J., Fernández-Fernández, M. R., Cambra, M., and García, J. A. 2000. Biotechnological aspects of plum pox virus. J. Biotechnol. 76:121-136.

26. Love, A. J., Laird, J., Holt, J., Hamilton, A. J., Sadanandom, A., and Milner, J. J. 2007. Cauliflower mosaic virus protein P6 is a suppressor of RNA silencing. J. Gen. Virol. 88:3439-3444.

27. Martínez, F., Lafforgue, G., Morelli, M. J., González-Candelas, F., Chua, N. H., Daròs, J. A., and Elena, S. F. 2012. Ultradeep sequencing analysis of population dynamics of virus escape mutants in RNAi-mediated resistant plants. Mol. Biol. Evol. 29:3297-3307.

28. Martínez-Priego, L., Donaire, L., Barajas, D., and Llave, C. 2008. Silencing suppressor activity of the Tobacco rattle virus-encoded 16-kDa protein and interference with endogenous small RNA-guided regulatory pathways. Virology 376:346-356.

29. Nicaise, V., Gallois, J. L., Chafiai, F., Allen, L. M., Schurdi-Levraud, V., Browning, K. S., Candresse, T., Caranta, C., Le Gall, O., and GermanRetana, S. 2007. Coordinated and selective recruitment of eIF4E and eIF4G factors for potyvirus infection in Arabidopsis thaliana. FEBS Lett. 581:1041-1046.

30. Niu, Q. W., Lin, S. S., Reyes, J. L., Chen, K. C., Wu, H. W., Yeh, S. D., and Chua, N. H. 2006. Expression of artificial microRNAs in transgenic Arabidopsis thaliana confers virus resistance. Nat. Biotechnol. 24:14201428.

31. Parizotto, E. A., Dunoyer, P., Rahm, N., Himber, C., and Voinnet, O. 2004. In vivo investigation of the transcription, processing, endonucleolytic activity, and functional relevance of the spatial distribution of a plant miRNA. Genes Dev. 18:2237-2242.

32. Pflieger, S., Blanchet, S., Camborde, L., Drugeon, G., Rousseau, A., Noizet, M., Planchais, S., and Jupin, I. 2008. Efficient virus-induced gene silencing in Arabidopsis using a 'one-step' TYMV-derived vector. Plant J. 56:678-690.

33. Qu, J., Ye, J., and Fang, R. 2007. Artificial microRNA-mediated virus resistance in plants. J. Virol. 81:6690-6699.

34. Rizzo, T. M., and Palukaitis, P. 1989. Nucleotide sequence and evolutionary relationships of cucumber mosaic virus (CMV) strains: CMV RNA 1. J. Gen. Virol. 70:1-11.

35. Schoelz, J. E., and Shepherd, R. J. 1988. Host range control of cauliflower mosaic-virus. Virology 162:30-37.

36. Schwab, R., Ossowski, S., Riester, M., Warthmann, N., and Weigel, D. 2006. Highly specific gene silencing by artificial microRNAs in Arabidopsis. Plant Cell 18:1121-1133.

37. Sokal, R. R., and Rohlf, F. J. 1995. Pages 730-736 in: Biometry, 3rd ed. W. H. Freeman \& Co., New York.

38. Voinnet, O. 2009. Origin, biogenesis, and activity of plant microRNAs. Cell 136:669-687.

39. Whitlock, M. C., and Schluter, D. 2009. Pages 156-157 in: The Analysis of Biological Data. Roberts \& Co., Greenwood Village, CO.

40. Zeng, Y., Wagner, E. J., and Cullen, B. R. 2002. Both natural and designed micro RNAs can inhibit the expression of cognate mRNAs when expressed in human cells. Mol. Cell 9:1327-1333.

41. Zhang, X., Li, H., Zhang, J., Zhang, C., Gong, P., Ziaf, K., Xiao, F., and Ye, Z. 2011. Expression of artificial microRNAs in tomato confers efficient and stable virus resistance in a cell-autonomous manner. Transgenic Res. 20:569-581.

42. Zhang, X., Yuan, Y. R., Pei, Y., Lin, S. S., Tuschl, T., Patel, D. J., and Chua, N. H. 2006. Cucumber mosaic virus-encoded 2b suppressor inhibits Arabidopsis Argonaute1 cleavage activity to counter plant defense. Genes Dev. 20:3255-3268. 